\title{
Magnetoencephalographic (MEG) brain activity during a mental flexibility task suggests some shared neurobiology in children with neurodevelopmental disorders
}

\author{
Alexandra Mogadam 1,2, Anne E. Keller ${ }^{2,3}$, Paul D. Arnold ${ }^{4,5}$, Russell Schachar ${ }^{2,6}$, Jason P. Lerch ${ }^{2,7,8}$, \\ Evdokia Anagnostou ${ }^{1,2,9}$ and Elizabeth W. Pang ${ }^{1,2,3^{*}}$ (D)
}

\begin{abstract}
Background: Children with neurodevelopmental disorders (NDDs) exhibit a shared phenotype that involves executive dysfunctions including impairments in mental flexibility (MF). It is of interest to understand if this phenotype stems from some shared neurobiology.

Methods: To investigate this possibility, we used magnetoencephalography (MEG) neuroimaging to compare brain activity in children ( $n=88 ; 8-15$ years) with autism spectrum disorders (ASD), attention deficit hyperactivity disorder (ADHD) and obsessive-compulsive disorder (OCD), as they completed a set-shifting/mental flexibility task.
\end{abstract}

Results: Neuroimaging results revealed a similar parietal activation profile across the NDD, groups suggesting a link to their shared phenotype. Differences in frontal activity differentiated the three clinical groups. Brain-behaviour analyses showed a link with repetitive behaviours suggesting shared dysfunction in the associative loop of the corticostriatal system.

Conclusion: Our study supports the notion that NDDs may exist along a complex phenotypic/biological continuum. All NDD groups showed a sustained parietal activity profile suggesting that they share a strong reliance on the posterior parietal cortices to complete the mental flexibility task; future studies could elucidate whether this is due to delayed brain development or compensatory functioning. The differences in frontal activity may play a role in differentiating the NDDs. The OCD group showed sustained prefrontal activity that may be reflective of hyperfrontality. The ASD group showed reduced frontal activation suggestive of frontal dysfunction and the ADHD group showed an extensive hypoactivity that included frontal and parietal regions. Brain-behaviour analyses showed a significant correlation with repetitive behaviours which may reflect dysfunction in the associative loop of the corticostriatal system, linked to inflexible behaviours.

Keywords: ASD, ADHD, OCD, MEG, Executive function, Set shifting, TOCS, RBS-R, Corticostriatal projections, Neurodevelopmental disorders

\footnotetext{
* Correspondence: elizabeth.pang@sickkids.ca

${ }^{3}$ Division of Neurology, Hospital for Sick Children, 555 University Avenue,

Toronto M5G 1X8, Canada

Full list of author information is available at the end of the article
}

(c) The Author(s). 2019 Open Access This article is distributed under the terms of the Creative Commons Attribution 4.0 International License (http://creativecommons.org/licenses/by/4.0/), which permits unrestricted use, distribution, and reproduction in any medium, provided you give appropriate credit to the original author(s) and the source, provide a link to the Creative Commons license, and indicate if changes were made. The Creative Commons Public Domain Dedication waiver (http://creativecommons.org/publicdomain/zero/1.0/) applies to the data made available in this article, unless otherwise stated. 


\section{Introduction}

Neurodevelopmental disorders (NDDs) are a heterogeneous group of disorders, characterized by compromised central nervous system development and aberrant brain function [1-3]. The most common NDDs include autism spectrum disorders (ASD), attention deficit hyperactivity disorder (ADHD) and paediatric obsessive-compulsive disorder (OCD). While each of these NDDs has its own distinct clinical phenotype (i.e. social communication impairments and repetitive behaviours in ASD, dysfunctions in attention regulation and hyperactivity in ADHD, and impaired control of obsessive thoughts and behaviours in OCD [1]); they are often co-morbid [4] and share genetic [5-7], neurobiological [8] and cognitive-behavioural characteristics, such as impairments in social perception [9], rigidity and difficulties with attention.

One cognitive characteristic observed in all three groups is that of impaired executive functions, including mental flexibility (MF). Mental flexibility comprises the ability to alter behavioural and thought patterns in response to environmental changes $[10,11]$ and is essential for adapting to changing surroundings, navigating social interactions and learning in academic and work environments. This crucial cognitive function can be assessed using a set-shifting task, in which participants are asked to match stimuli, with matching criteria shifting every few trials.

Mental flexibility relies on a network of brain regions spanning prefrontal, posterior parietal and insular regions, the basal ganglia and anterior cingulate cortex (ACC; [11-13]), as well as the temporal pole (TP) and pre- and supplementary motor regions in typically developing children (TDC; [14]). Functional neuroimaging studies have investigated the neural correlates of MF in children with ASD [15-17] and adolescents and adults with ASD [18], ADHD [19-21], and OCD [19, 22]. In comparison with TDC, these studies have found differences in brain activity associated with MF within these groups, with one reporting similarities across ADHD and OCD [19], although none have compared all three groups together. Similarly, a diffusion tensor imaging (DTI) study of ASD, ADHD and OCD suggested that there may also be shared structural deficits in all three groups, with ASD and ADHD additionally affected [8]. Together, this growing body of neurobiological and genetic evidence proposes that these NDDs are not separate entities that sometimes co-occur, but in fact, are perhaps part of a spectrum with shared aetiologies and overlapping phenotypes (as discussed by [2]).

Most studies of MF have used fMRI as their method of choice. While this tool is excellent for spatial investigations, it is more limited in its temporal resolution [23]. Magnetoencephalography (MEG) is a neurophysiological modality which tracks neural activity with millisecond accuracy making it an excellent complement to fMRI [24]. Investigations into the fast-paced temporal dimension of brain activity can significantly contribute to our understanding of the dynamics of cognitive processes such as MF, in both typical and non-typical populations. In light of emerging evidence of disrupted connectivity (as measured by high temporal resolution oscillatory synchronization) across multiple areas in the brain during tasks of MF in children with ASD [15, 17, 25], we decided to employ MEG in our investigation of MF in NDDs.

To investigate the neural bases of mental (in) flexibility in children with NDDs, we recruited children with ASD, ADHD and OCD to complete a set-shifting task in the MEG scanner. As there is increasing research suggesting that NDDs may exist along a continuum, we hypothesized that the common behavioural manifestation of cognitive inflexibility across ASD, ADHD and OCD groups may be due to some shared underlying neural substrates, existing along a spectrum. Specifically, based on Ameis et al.'s DTI research, we expected to find greater similarities in activity in ASD and ADHD groups, with both groups more affected than the OCD group.

\section{Materials and methods Participants}

We recruited 116 children with an NDD between the ages of 8-15 years. After data cleaning for artefacts, a total of 88 children (38 ASD, 28 ADHD, 22 OCD) were included in our final analyses (see Table 1 for demographics). Participants were recruited through the Province of Ontario Neurodevelopmental Disorders (POND) Network from clinics at the Holland Bloorview Kids Rehabilitation Hospital (ASD) and the Hospital for Sick Children (SickKids; ADHD and $\mathrm{OCD}$ ) in Toronto. Inclusion criteria were a primary clinical diagnosis of ASD, ADHD or OCD, normal or corrected-to-normal vision, ability to comply with neuroimaging protocols and no contradictions for neuroimaging. Co-morbidities and psychotropic medication use were noted but not excluded.

Upon enrolment, primary clinical diagnoses were confirmed using disorder-specific diagnostic measures: Autism Diagnostic Observation Schedule-2 (ADOS; [30]) and Autism Diagnostic Interview-Revised (ADI-R; [31]) for ASD, Parent Interview for Child Symptoms (PICS; [26]) for ADHD, and the Child Yale-Brown Obsessive Compulsive Scale (CYBOCS; [27]) for OCD.

\section{Neuropsychological assessments}

Full-scale intelligence quotients (FSIQ-2/4: WISC-IV ${ }^{\oplus}$, WASI-I/-II ; Full IQ: Stanford Binet Intelligence Scales ${ }^{\odot}$ ) and four parent-questionnaires were administered to measure repetitive (Repetitive Behaviour Scale-Revised (RBS-R; $[28,29])$ ), obsessive-compulsive (Toronto Obsessive- 
Table 1 Summary of demographic information and neuropsychological assessments

\begin{tabular}{llll}
\hline & ASD $^{1}$ & ADHD & OCD $^{2}$ \\
\hline Number $(N=88)$ & 38 & 28 & 22 \\
Age & $12.26 \pm 2.19$ years & $12.13 \pm 1.89$ years & $11.58 \pm 2.29$ years \\
Male to female & $31: 7$ & $24: 4$ & $14: 8$ \\
FS-IQ-4/SB-IQ & $99 \pm 18(n=36)$ & $98 \pm 17(n=22)$ & $117 \pm 18(n=8)$ \\
RBS-R total $^{3}$ & 30 & 13 & 30 \\
SWAN-inattention $^{4}$ & 5 & 6 & 3 \\
SWAN-hyperactivation $^{4}$ & 4 & 4 & 2 \\
TOCS $^{5}$ & -7 & -26 & 18 \\
\hline
\end{tabular}

${ }^{1} 28.57 \%$ (10/35) of participants with ASD received a secondary diagnosis of ADHD

${ }^{2} 27.27 \%(6 / 22)$ of participants with OCD received a secondary diagnosis of ADHD and $4.55 \%(1 / 22)$ of participants with OCD received a secondary diagnosis of ASD

${ }^{3}$ Repetitive Behaviour Scale-Revised (total score and number of endorsed items score) $[26,27]$

${ }^{4}$ Strengths and Weakness of ADHD Symptoms and Normal Behaviour Rating Scales (inattention and hyperactive sub-measures) [28]

${ }^{5}$ Toronto Obsessive-Compulsive Scale [29]

Compulsive Scale (TOCS; [32])) and inattentive and hyperactive [Strengths and Weaknesses of ADHD Symptoms and Normal Behaviour Rating Scales (SWAN, inattention and hyperactive sub measures; [33])] behavioural patterns in participants. See Table 1 for group scores.

\section{Task}

To assess MF in our clinical groups, we employed an MEG-compatible Intra-Extra Dimensional Set Shift task (IED-task) previously used in our group to test adults [34] and children [14]. In this task, participants match a target stimulus based on a matching rule that changes every few trials. Participants are required to 'shift' to the new rule to have a correct match. There were two types of shifts in our study, 'extra-dimensional' and 'intradimensional', where the former involves a more difficult shift between categories (dimensions), while the latter involves an easier shift, within categories. As the extradimensional shift is more difficult, it better taps into the mental processes involved in set-shifting; thus, we present the extra-dimensional results only. From here, this is referred to as the 'Shift' condition. See Additional file 1, Section 1-1 for full details.

\section{Behavioural analyses}

Accuracy and reaction time for correct Shift and Non-Shift trials were compared across groups using a linear fixed-effects model in SPSS ${ }^{\circ}$ (v24), with repeated measures for shift type, age as a covariate and an unstructured repeated covariance type.

\section{Imaging data acquisition and pre-processing}

MEG data were acquired supine in a 151-channel CTF Omega system (MISL, Coquitlam, Canada). Analyses were conducted using SPM12 [35] and FieldTrip [36]. Data were filtered $(1-50 \mathrm{~Hz})$ and epoched $(-500-1500$ $\mathrm{ms})$. Artefacts were rejected ( $>2500 \mathrm{fT}$ ) and removed (heart and eye artefacts) using ICA [37, 38]. The data were then averaged, and root mean square (RMS) activity plots, summed over all MEG channels, across time, were generated. See Additional file 1, Sections 1-2 and 1-3 for more imaging and pre-processing details, respectively.

\section{MEG analyses}

Empirical Bayesian beamforming (EBB; [39, 40]) was applied to reconstruct sources (12-mm FWHM Gaussian kernel smoothing) from 50 to $500 \mathrm{~ms}$ post-stimulus onset, with sliding overlapping time windows $(100 \mathrm{~ms}$ wide, $50 \mathrm{~ms}$ overlap), resulting in a total of eight windows of interest (i.e. $50-150,100-200 \mathrm{~ms}$, etc.). Between- and within-group contrasts were conducted using independent samples $t$ test [SPM(T)], corrected for multiple comparisons with a modified Bonferroni applied to the $p$ value of 0.05 [41]. All results report significant corrected brain activity $\left(p_{\text {corr }}<0.05\right)$ which was visualized through MRIcron [42].

We first conducted a within-group analysis where, for each group, we used a multifactorial design [43] to contrast the Shift with the Non-Shift condition to identify the brain activity associated with shifting. This generated, for each group, a list of regions with significantly greater activation for the Shift condition within each time window.

To explore differences between groups, a multifactorial design [43] was used to contrast the images based on our hypotheses generated from the literature [8]. We tested the following Shift contrasts: OCD > ASD, OCD > ADHD, ADHD > ASD, and ASD > ADHD.

\section{Brain-behaviour analyses}

To further probe the cross-diagnosis shared neurobiological correlates of mental inflexibility, we investigated brain-behaviour relations collapsed across the group. We 
used a linear regression model (controlled for age) to test whether the magnitude and/or latency of brain activity predicted behavioural measures (TOCS, RBS-R and SWAN), regardless of clinical group (see Additional file 1, Section 1-4). The TOCS and RBS-R measure obsessive-compulsive and repetitive behaviours, respectively, and the SWAN measures inattentive and hyperactive behaviours. These scales were selected as they quantify the severity of behavioural symptoms that may reflect, and/or contribute to, mental inflexibility.

\section{Results}

\section{Behavioural results}

For accuracy, a significant main effect was observed for 'Age' $[F(1,82)=4.187, p=0.044]$ but not for 'Group' nor 'Shift Type'. There were no significant interactions. The average age-adjusted accuracy scores per trial type, for each group, are contained in Table 2 (upper).

For reaction time, the fixed effects model revealed a main effect for 'Shift Type' $[F(2,82)=7.050, p=0.001]$ only. Post hoc analyses, adjusted for multiple comparisons using the Bonferroni method, revealed that NonShift trials were faster than Shift trials $[p<0.001]$. There were no significant main effects for 'Group' and no significant interactions. The mean age-adjusted reaction times by group and trial type can be found in Table 2 (lower).

\section{MEG results}

\section{Within-group source level analyses: brain regions involved} in set-shifting

Spatiotemporal activity plots identifying brain regions that were significantly more active during set-shifting, for each group, are contained in Fig. 1. For the ASD group, there was sustained activity in parietal lobes involving both inferior and superior lobules (BA 7, 39, 40), with contributions from both hemispheres. Prefrontal activity was dominated by the right inferior frontal gyrus (IFG; BA 44, 45, 47), starting $200 \mathrm{~ms}$ post-stimulus onset, until $450 \mathrm{~ms}$. Additional activity was found in the right temporal lobe (BA 37, 38) and the left parahippocampus (BA 36) from 150 to $350 \mathrm{~ms}$, as well as in the right pre- and supplementary motor areas (BA 6) from 250 to $350 \mathrm{~ms}$.

In the ADHD group, similar to the participants with ASD, parietal activity was sustained, although the activity was predominantly in the left hemisphere, in both inferior and superior lobules (BA 7, 39, 40). The right prefrontal regions (BA 10) were briefly active from 50 to $150 \mathrm{~ms}$, and then again later from 250 to $450 \mathrm{~ms}$ bilaterally in inferior, middle and superior frontal gyri (BA 8, $10,46,47)$. Similar to ASD, additional activity was found in pre- and supplementary motor areas (BA 6) and the temporal lobe (BA 37), though in the contralateral hemisphere.

Finally, children with OCD also displayed early and sustained bilateral activity in the parietal lobes (BA 7, 39,40 ) from 50 to $400 \mathrm{~ms}$ post-stimulus onset. Frontal activity was similarly sustained from 50 to $500 \mathrm{~ms}$, also in both hemispheres, across inferior, middle and superior frontal gyri (BA 9, 10, 11, 44, 46, 47). Bilateral pre- and supplementary motor areas (BA 6) were also active early on, with the temporal lobe (BA 37, 38) displaying activity somewhat later, predominantly in the left hemisphere.

\section{Between-group contrasts}

As per our a priori hypotheses, we conducted four between-group comparisons (contrasting the Shift conditions of two groups at a time; $p_{\text {corr }}<0.05$ ) where significant differences are displayed in Fig. 2 and delineated in Additional file 1: Table S1. Our a priori hypotheses were that the OCD group would show greater activations compared to both ASD and ADHD groups. The OCD $>$ ASD contrast (Fig. 2a) revealed significantly greater and significantly more sustained (50-350 ms) activity, mainly in frontal regions (middle frontal gyrus, MFG; BA 10) for the OCD group. As well, the OCD group showed a brief $(100-200 \mathrm{~ms})$ period of greater activity in the right

Table 2 Accuracy and reaction times for the set-shifting task, by clinical group

\begin{tabular}{|c|c|c|c|c|c|c|}
\hline & \multicolumn{2}{|c|}{ Non-shift } & \multicolumn{2}{|c|}{ Intradimensional (easy) shift } & \multicolumn{2}{|c|}{ Extradimensional (hard) shift } \\
\hline & Mean & $\overline{\mathrm{SE}}$ & Mean & SE & Mean & SE \\
\hline \multicolumn{7}{|l|}{ Accuracy } \\
\hline ASD & $93.7 \%$ & 0.8 & $93.4 \%$ & 1.1 & $91.7 \%$ & 1.2 \\
\hline ADHD & $94.2 \%$ & 0.9 & $93.2 \%$ & 1.3 & $89.7 \%$ & 1.4 \\
\hline OCD & $93.9 \%$ & 1.0 & $92.7 \%$ & 1.5 & $90.8 \%$ & 1.6 \\
\hline \multicolumn{7}{|c|}{ Reaction time } \\
\hline ASD & $755 \mathrm{~ms}$ & 41 & $834 \mathrm{~ms}$ & 60 & $914 \mathrm{~ms}$ & 43 \\
\hline ADHD & $812 \mathrm{~ms}$ & 48 & $912 \mathrm{~ms}$ & 70 & $948 \mathrm{~ms}$ & 50 \\
\hline OCD & $773 \mathrm{~ms}$ & 55 & $865 \mathrm{~ms}$ & 80 & $870 \mathrm{~ms}$ & 58 \\
\hline
\end{tabular}




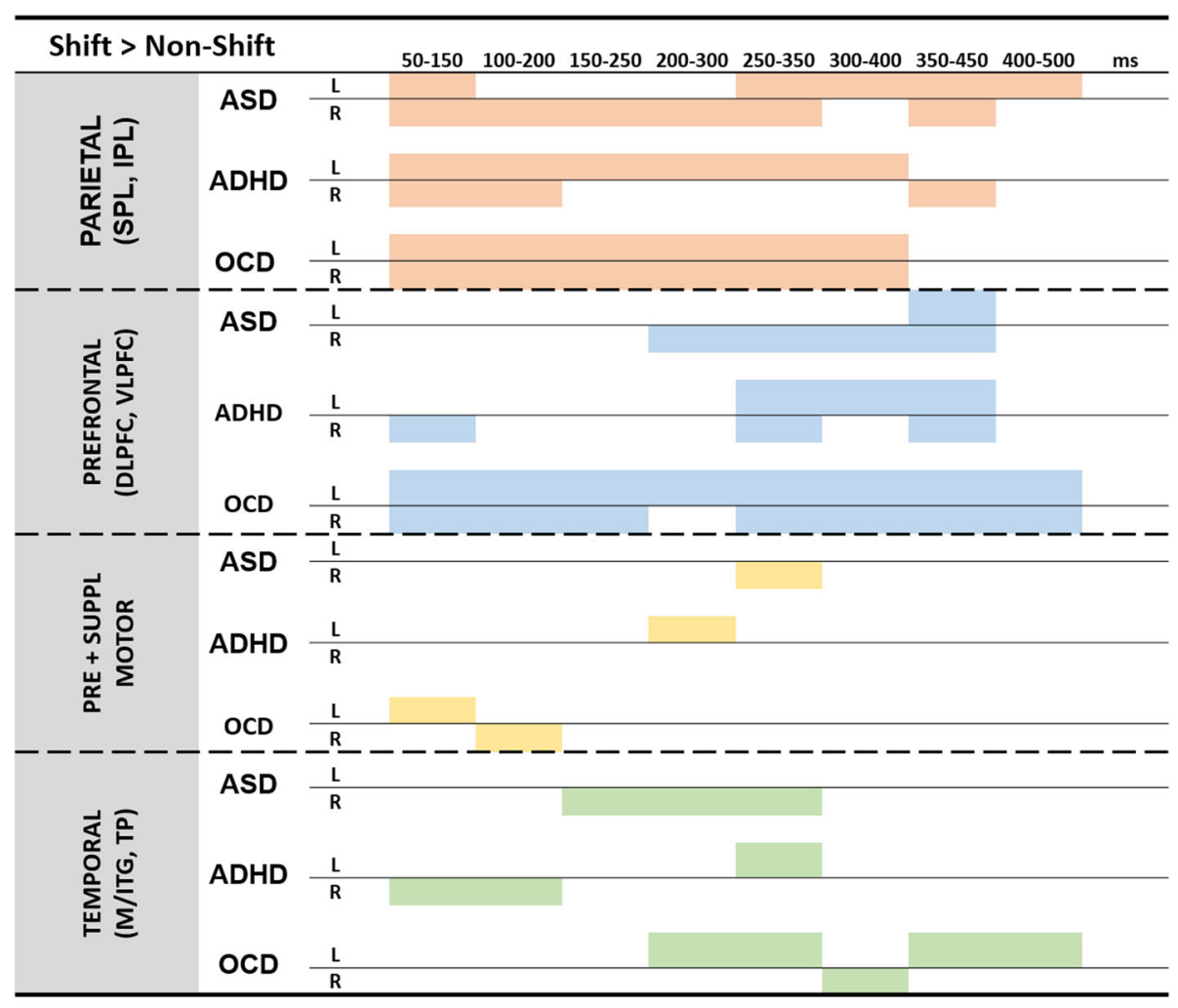

SPL: superior parietal lobule; IPL: inferior parietal lobule; DLPFC: dorsolateral prefrontal cortex; VLPFC: ventrolateral prefrontal cortex; M/ITG: middle/inferior temporal gyrus; TP: temporal pole

Fig. 1 Individual brain activity profiles within each clinical group. For each brain region, the significant activations $\left(p<0.05_{\text {corr }}\right)$ associated with set-shifting are shown, for each clinical group. The activity profiles reveal a shared pattern of sustained parietal activity in all three groups, late and limited prefrontal activity in ASD and ADHD, and sustained frontal activity in OCD. Other regions (pre- and supplemental motor cortices and temporal regions) do not show similarities across groups. SPL superior parietal lobule, IPL inferior parietal lobule, DLPFC, dorsolateral prefrontal cortex, VLPFC ventrolateral prefrontal cortex, M/ITG middle/inferior temporal gyrus, TP temporal pole

superior temporal gyrus (STG; BA 22) and the left ACC (BA 25). The OCD > ADHD contrast (Fig. 2b) showed more between-group differences with greater and significantly more sustained (50-300 ms) activity in bilateral prefrontal regions (MFG/ IFG; BA 10, 11, $45,46,47)$ for OCD. Additional differences were found in the parietal (right angular gyrus; BA 39; $50-150 \mathrm{~ms}$ ) and temporal regions (right STG/BA 22; $100-200 \mathrm{~ms}$ and left TP/BA 38; 250-350 ms).

Because we hypothesized that the ASD and ADHD groups would be more similar, we conducted contrasts in both directions. For the ASD > ADHD (Fig. 2c) contrast, a few differences were observed with the ASD group showing greater activity in the right parietal (supramarginal gyrus, SMG/BA 40; 50$150 \mathrm{~ms}$ and superior parietal lobule, SPL/BA 7; 150$300 \mathrm{~ms}$ ) and left temporal (TP/BA 38; 150-250 ms) regions. In the other direction, ADHD $>$ ASD (Fig. 2d), the differences were very sparse. The ADHD group showed greater activations in a late time window in the right IFG/BA 47 (300-450 ms) and right STG/BA 22 (300-500 ms).

\section{Brain-behaviour analyses}

To explore potential relationships between brain regions involved in MF and continuous behavioural measures of clinical symptomology, we regressed peak latency and magnitude during Shift trials with measures of obsession-compulsion (TOCS), repetitive behaviours (RBS-R) and attention/hyperactivity (SWAN), controlling for age. Only the first two measures (TOCS and RBS-R) showed significant relationships (Table 3).

For the measure of obsessive-compulsive behaviours captured by the TOCS, we found that the peak latency in two frontal regions, the right superior frontal gyrus (SFG) $\left[F(2,79)=4.084, p=0.021\right.$; adjusted $R^{2}=0.071$; $B=-0.102, p=0.008]$ and left IFG pars triangularis $[F(2$, 79) $=3.419, p=0.038$; adjusted $R^{2}=0.056 ; B=-0.086, p=$ $0.015]$, was negatively related with TOCS score. That is, 


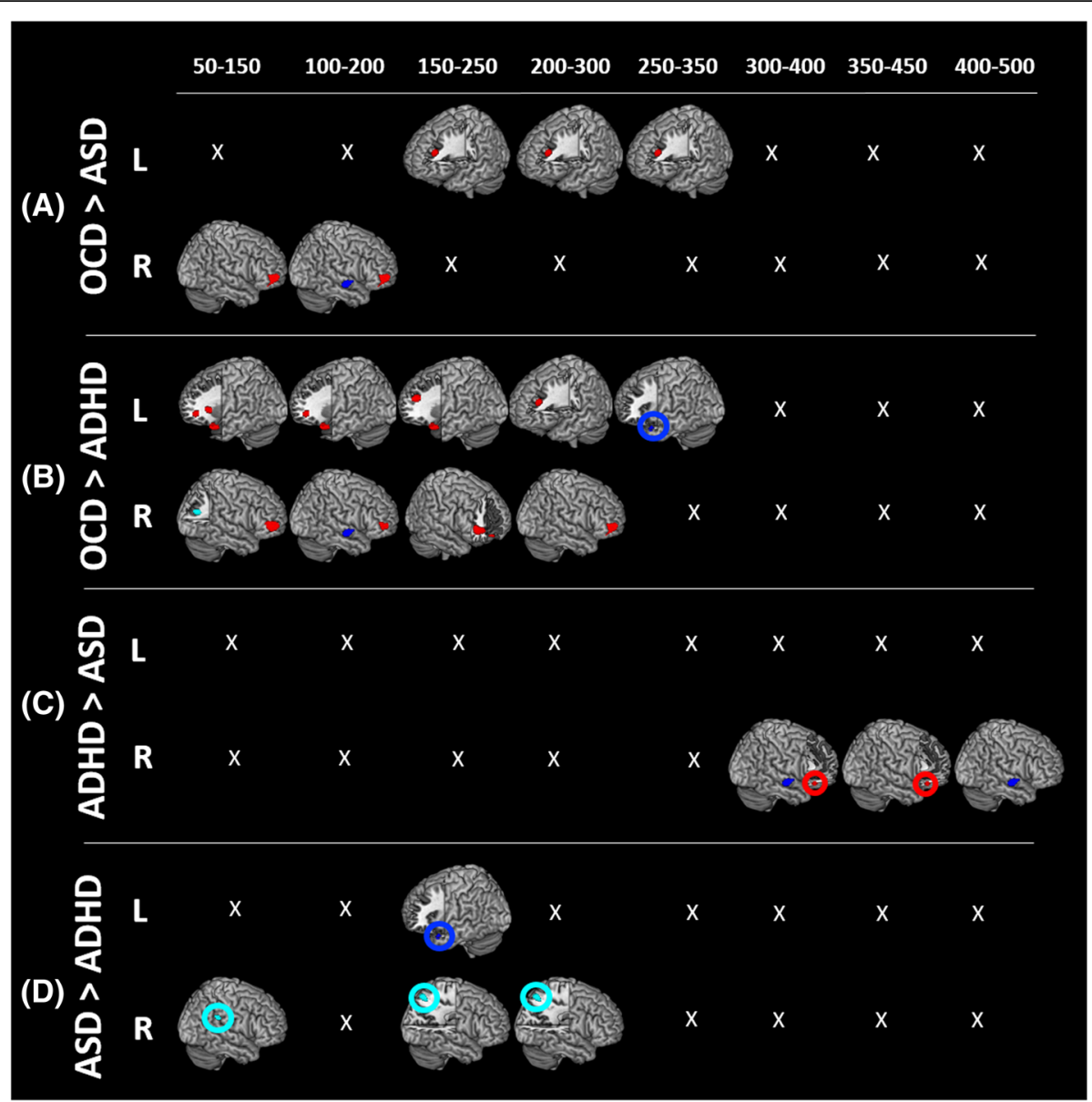

frontal temporal parietal

Fig. 2 Between-group contrast results, $p=0.05_{\text {corr }}$. Between-group contrasts of brain activity associated with mental flexibility (MF). The first two contrasts, $\mathbf{a} O C D>A S D$ and $\mathbf{b} O C D>A D H D$, reveal significantly greater bilateral prefrontal activity in OCD, compared to both ASD and ADHD. The $\mathbf{c} A D H D>A S D$ and $\mathbf{d} A S D>A D H D$ contrasts show fewer differences, with ASD showing greater parietal activity and reduced frontal activity compared to ADHD

faster peak latencies in these two frontal regions were associated with higher scores, or greater morbidity, regardless of clinical group, on the TOCS scale.

For the measure of repetitive behaviours, we found that a significant linear regression equation predicted RBS-R total scores based on peak power values, extracted during peak two $(150-300 \mathrm{~ms}),[F(2,79)=$ 4.059, $p=0.021$; adjusted $\left.R^{2}=0.070\right]$. Peak power values in the left angular gyrus were significantly positively related to RBS-R total scores, $[B=72.025$, $p=0.008]$, indicating increased power/activation was associated with greater RBS-R total scores or greater morbidity.

\section{Discussion}

In this study, we used MEG to investigate neural processing involved in a MF task in children with ASD, ADHD and OCD. The ease with which participants completed the task (there were no group differences, with accuracy at approximately 90\%) indicated that the children were able to set-shift successfully.

Table 3 Significant correlations between brain-behaviour measures

\begin{tabular}{llll}
\hline Behavioural measure & Brain region & Brain measure & $\beta$ (beta) \\
\hline TOCS $^{1}$ & Right SFG & Peak latency & -0.299 \\
TOCS & Left IFG & Peak latency & -0.274 \\
RBS-R (total) & Left angular gyrus & Amplitude & 0.008 \\
\hline
\end{tabular}

\footnotetext{
${ }^{1}$ Toronto Obsessive-Compulsive Scale
}

${ }^{2}$ Repetitive Behaviour Scale-Revised (total score) 
Similar regions underlie mental flexibility in all groups In both adults [34] and typically developing control children (TDC; [14]), MF processing has been shown to be subsumed by hubs in bilateral fronto-parietal cortices, the insula and the ACC, with children drawing on additional premotor and temporal lobe regions. Our results concur with this literature as we found that the NDD groups showed recruitment of these brain regions during the task.

\section{Overlapping activation patterns observed in all groups}

The literature in both adults [34] and children [14] suggests that parietal and frontal hubs activate sequentially, without overlap, when completing a set-shifting task. In the current study, our NDD groups showed extensive overlap in the activation pattern of parietal and frontal regions (Fig. 1), with a distinct absence of the sequential progression of activation that is described in the literature. Instead, all three NDD groups showed sustained SPL and inferior parietal lobule (IPL) activation throughout the processing of the task. This sustained posterior parietal activity suggests that all three clinical groups share a strong reliance on the posterior parietal cortices to complete the MF task.

According to the posterior-to-anterior theorem of brain development $[44,45]$, we know that parietal gyri develop before frontal regions and that often, the posterior parietal cortices play a larger role in mediating executive functions in childhood, until, with increasing age, the frontal areas become more developed and can assume their role in processing executive functions [46-49]. We speculate that our observation of sustained parietal activation that overlaps with the timing of frontal activations suggests that children with NDDs need greater assistance from the parietal regions for their executive functioning. Future studies should investigate whether this observation of high reliance on parietal regions is indicative of delayed brain development or compensation for prefrontal dysfunction.

\section{Prefrontal activation pattern differentiates $O C D$}

While the NDD groups all showed a similar pattern of sustained parietal activity, the pattern of frontal activations was significantly different between groups, suggesting that this may be a distinguishing feature. Based on the pattern of findings by Ameis et al. in the same NDDs, and in line with our within-group results (Fig. 1), we expected between-group analyses to show the OCD group to less similar to the other two, and we expected ASD and ADHD groups to be more comparable to each other.

Our between-group analyses $(\mathrm{OCD}>\mathrm{ASD}$ and $\mathrm{OCD}>$ ADHD) revealed that children with ASD and ADHD display significantly decreased activity in prefrontal regions compared to children with OCD. The OCD > ADHD contrast revealed the greatest differences, with ADHD showing bilaterally reduced frontal activity across the inferior and middle frontal gyri. These findings are consistent with other functional neuroimaging studies in ADHD which have shown hypofrontality in MF, as well as executive functions more broadly [19-21, 50-54].

The sustained prefrontal activity in OCD may be reflective of hyperfrontality, a functional characteristic that has previously been reported in the OCD literature ([55], for reviews, please refer to [56, 57]), although not necessarily in relation to MF. In adults with OCD, fMRI studies show reduced activation in the classic functional hubs of mental flexibility [58], which related to performance [59]. We were able to hold performance constant between groups; thus, our increased activation may be suggestive of a compensatory mechanism recruited to maintain this high function. Only one study has been conducted in children with OCD [22]. Using fMRI and a set-shifting task, this study reported a decreased hemodynamic response in the left IFG in children with OCD; however, they report greater grey matter density in dorsolateral prefrontal cortex (BA 10), IFG and ACC [22], which may be the neurophysiological mechanism underlying our observation of increased activation in this area. In general, our findings concur with the idea of dysregulation in the prefrontal regions in OCD; however, further studies are needed to understand whether atypical activations are symptomatic of dysfunctional processing or indicative of compensatory function.

\section{ASD and ADHD differentiated by frontal/parietal abnormalities}

We did not have a priori hypotheses as to how the ASD and ADHD groups would compare with each other; thus, we conducted our comparisons in both directions. As we would have predicted from Fig. 2, fewer differences were found in the ASD > ADHD and ADHD > ASD contrasts, compared to the contrasts with the OCD group. However, a definite pattern emerged where we observed significantly reduced right parietal activation in ADHD and reduced right frontal activation in ASD.

In the ADHD group, this reduced parietal activation compared to ASD, and the reduced frontal activation compared to OCD (described above), is consistent with many studies showing that individuals with ADHD have extensive hypoactivity that includes the frontal lobes and the parietal regions, as well as the striatum, insula and ACC [19-21, 51-53].

In ASD, previous studies have shown dysregulations in the connectivity and synchrony of brain regions/networks involved in MF [25], as well as an increased reliance on the parietal lobes in set-shifting compared to 
TDC $[15,17,60]$. It has been suggested that the brains of individuals with ASD do not develop efficient longrange cortical connections during development, and at the same time, have very well-established short-range connections (U-fibres), resulting in hyper-connectivity of local brain hubs [61-64]. Such a structural organization of the brain would show excessive activation of interparietal cortical networks and result in altered function, as in the case of MF. Because of the disruptions in the efficiency of long-range connections, it is possible that the ASD group cannot efficiently recruit their frontal regions, and therefore exercise a greater reliance on their parietal lobes for successful task execution.

\section{Brain-behaviour analyses}

For our first set of MEG analyses (featuring the withinand between-group neuroimaging contrasts), we grouped participants according to their primary diagnosis. While our MEG contrasts did reveal unique MF group-specific brain activity profiles, we also found striking similarities across the NDDs. Increasingly, there is evidence that NDDs are not separate entities that sometimes co-occur, but in fact, may be part of a spectrum with shared aetiologies and overlapping phenotypes [2]. To this end, we conducted brain-behaviour correlations, across all participants, to explore whether measures of neuromagnetic activity in brain regions involved in setshifting predicted inflexibility-related symptom severity, irrespective of diagnostic group.

We used the RBS-R, TOCS and SWAN as measures of severity of symptomatic behaviours reflective of, and/or contributing to, mental inflexibility. For both the RBS-R and the TOCS, we found that brain activity predicted symptom severity, albeit in opposite directions. Bilaterally in the dorsolateral prefrontal cortices (right SFG and left IFG), we found a negative relation between peak latency and TOCS scores, such that faster peak latencies were significantly related to poorer (increased) TOCS scores. As expected, the OCD group had the greatest morbidity on the TOCS scale. There are a few different ways that we could interpret this finding. It is likely that this brain-behaviour relation is tied to the earlier and generally greater frontal activity observed in the OCD cohort during the MF task. Consistent with the literature, these findings may suggest that the prefrontal regions are hyperactive in children with OCD-like symptoms ([55] for reviews, please refer to $[56,57])$. Another possibility is that this brain-behaviour relation indicates that children with NDDs who activate their prefrontal regions earlier exhibit behaviours associated with OCD symptomology, such as impulsive/compulsive behaviours or hyper-performance monitoring. The latter is supported by a study reporting hyperactivity in the MFC during performance monitoring in children with OCD, suggesting greater error-monitoring and a general excessive concern to perform well in this clinical group [55].

In addition to the finding in the prefrontal regions, we also found a significant brain-behaviour relation in the left angular gyrus of the parietal lobe. Increased peak power was positively related with total scores on the RBS-R, meaning that increased peak power was associated with increased repetitive behaviour morbidity. These findings would suggest that increased activity in the left parietal region, a key hub for set-shifting functions, would be associated with increased morbidity of repetitive behaviours. Overall, these brain-behaviour results fit with the between-group comparisons, implying hyperactivity in parietal regions across NDD groups during an MF task. We further speculate that the more a child with an NDD relies on this pronounced involvement/activation of posterior parietal regions during such tasks, the greater their mental inflexibility. Neuropsychological research in adults and children with ASD examining error type on mental flexibility tasks has found a correlation between regressive errors on the task and repetitive behaviour symptomology $[65,66]$. While the participants in our study showed extremely high performance, future neuroimaging studies utilizing more challenging tasks could assess atypical brain activity, its interaction with symptomology, error and performance functioning.

In a review paper examining the neurobiology of repetitive behaviours, Langen et al. discuss the different ways in which disruptions to the corticostriatal projections can contribute to inflexible behaviours. Corticostriatal projections are white matter tracts that project from the cortex to the striatum of the basal ganglia, a structure important for motor function and more generally goal-directed behaviour; disruptions to these projections have been associated with repetitive, restrictive and rigid behaviours in various disorders [67, 68]. Repetitive behaviour types can be classified according to their neuroanatomical substrates, and thus, mapped onto distinct corticostriatal loops [69]. Of particular interest is the associative loop, which consists of prefrontal and posterior parietal projections to the striatum, including from the regions in which we found significant brainbehaviour relations $[67,70,71]$. Langen et al. propose that dysfunctions in the associative loop may present behaviourally as impulsivity and/or rigidity. In light of this, it is possible that our brain-behaviour findings are reflective of an underlying corticostriatal dysfunction, impacting the ability of the involved neural regions to operate efficiently, including those that are important in set-shifting, resulting in symptoms of greater inflexibility.

The other corticostriatal loops are thought to be involved with other forms of inflexible behaviour, with the 
sensorimotor circuit associated with (atypical) stereotypical motor behaviour, and the limbic circuit with motivation-associated facets of behaviour and obsessions and compulsions [69]. Different combinations of variations or pathologies in these loops can create unique and inflexible behavioural patterns across individuals, creating the complexity and overlap in symptoms that are observed in disorders where inflexibility and repetitive behaviours are of issue [72]. Langen et al. propose that these behavioural patterns fall along a continuum, where, for example, a more pronounced abnormality in prefrontal or posterior parietal regions of the corticostriatal system may result in symptomology that would look more like the OCD or ASD cohorts, respectively. In this manner, a child with OCD who displays prefrontal over-engagement during a challenging MF exercise may perform below average, similar to a child with ASD, who displays less and later prefrontal engagement. Even if disruptions occur at different locations, or in different loops, as both children most likely have impairments in their corticostriatal projections, their behavioural output looks similar.

Research into the structural and functional health of frontostriatal regions in NDDs shows ubiquitous atypicalities. Children with ASD and ADHD show decreased white matter integrity along the corticostriatal tracts compared to controls [8]. Other studies have linked atypical frontostriatal (micro) structures to repetitive behaviours in ASD [73-75] and to more errors and more trials to complete a set-shifting task in ADHD [76]. A recent fMRI study in children with ASD and OCD found a relation between increased functional connectivity across frontostriatal regions during resting state and increased morbidity on their measure of repetitive behaviours [77], although this may reverse in adulthood [78].

To further explore the health and function of corticostriatal fibres in children with NDDs, future studies may consider taking a multi-model imaging approach, exploring white matter integrity of the loops and whether any correlations can be found with MF function. As well, it would be of interest to take a blind approach to the analysis and see whether primary diagnoses hold.

\section{Limitations}

There are some important limitations to consider. We are not able to draw direct comparisons between the NDD groups and typically developing children as there was not a control group in this study. Our previous work [14] in typically developing children used the identical task, acquisition parameters and processing pipeline of this study; however, there was an equally balanced sex ratio, whereas in the current study, the cohorts are male-dominated, as would be expected in NDDs [79-81]. Because of the increasing evidence that sex impacts the presentation of the NDDs, we chose not to directly compare the controls to the NDD groups. Future studies should target recruitment of additional males in the control group so as to maintain comparable sex ratios with the NDDs. A second limitation is that while comorbidities and medications were noted, they were not factored into the analysis. Finally, while we interpret our significant brain-behaviour results, it should be noted that these values account for only a small part of the variance. Future studies, possibly with much larger sample sizes and incorporating an age- and sex-matched control group, should attempt to address these limitations. Despite these limitations, we believe in the value of these findings and hope they may be hypothesis-generating for other groups working in this field.

\section{Conclusion}

In conclusion, while children with ASD, ADHD and OCD behaviourally share the same impairment in MF, using MEG, we found a pattern of similarities and differences in the neurobiological bases supporting this executive function. We observed that the three groups share neurofunctional characteristics in the parietal regions, but differ primarily in the frontal lobes. We observed that the NDD groups showed an absence of sequential brain activations, but instead, they showed sustained parietal activation which overlapped with frontal activation. This finding suggests that the three clinical groups share a delay or irregularity in brain development; a longitudinal study with a control group is recommended to draw firmer conclusions within this domain. ASD and ADHD groups seemed more affected than the OCD with limited and late frontal lobe activations; however, it remains to be seen whether the sustained prefrontal engagement in OCD is typical. Analyses linking brain activity with behavioural symptom measures revealed significant relations between the activity in prefrontal and parietal regions, and morbidity as measured on behavioural scales of repetitive and stereotypical behaviours, as well as obsessive-compulsive behaviours. These, in turn, may be reflective of a dysfunction in the associative loop of the corticostriatal system, which has been associated with inflexible behaviours, and has been found to be affected in children with NDDs. These findings raise the possibility that this neural system may be a target for intervention. Finally, we believe our findings are in line with new thinking that the NDDs exist along a complex continuum, where, despite the differing core phenotypic characteristics of the existing diagnostic groupings, NDDs appear to share some 'deeper' facets that become evident when probing genetic or neurobiological underpinnings of the disorders. 


\section{Additional file}

Additional file 1: Supplement 1. (PDF $212 \mathrm{~kb}$ )

\section{Acknowledgements}

The authors thank the many children and families involved in the study, as well as the many members of the POND recruiting, diagnosis, assessment and imaging teams who made this work possible. Additionally, we would like to thank Amanda Robertson, MyLoi Huynh, Olivia Dell'Unto, Julie Sato and Veronica Yuk who assisted with the study.

\section{Authors' contributions}

AM conducted the study and co-wrote the manuscript draft as part of her graduate thesis at the University of Toronto. AEK developed the neuroimaging analysis pipeline for this study and assisted with neuroimaging statistics. PDA consulted on the study design and recruited and oversaw the cohort of children with OCD. RS consulted on the study design, recruited and oversaw the cohort of children with ADHD. JPL helped to design the study protocol and neuroimaging details. EA helped to design the study, secured funding and recruited and oversaw the cohort of children with ASD. EWP supervised the study and co-wrote the manuscript draft with the first author. All authors contributed to the manuscript draft and approve its submission.

\section{Funding}

The MEG neuroimaging component of this work was completed as part of Alexandra Mogadam's graduate thesis and was supported by the Province of Ontario Neurodevelopmental Disorders (POND) Network, in partnership with the Ontario Brain Institute (OBI). Recruitment of the clinical groups is supported as follows: Paul D. Arnold holds the Alberta Innovates Health Solutions (AlHS) Translation Health Chair in Child and Youth Mental Health; Russell Schachar receives funding from DNA Genotek, Canadian Institutes of Health Research and TD Bank.

\section{Availability of data and materials}

The dataset supporting the conclusions of this article is available in the Brain-CODE repository (https://www.braincode.ca).

\section{Ethics approval and consent to participate}

The study was approved by the SickKids Research Ethics Board, and informed written consent and verbal assent were obtained from parents and children, respectively.

\section{Consent for publication}

All authors approve the publication of this manuscript.

\section{Competing interests}

Russell Schachar provides consultation to Highland Therapeutics/Ironshore Pharmaceuticals, has stock in ehave biotechnology company and previously was a consultant to Lilly Corp and Purdue Pharma. Evdokia Anagnostou provides consultation to Roche, receives in-kind support from AMO Pharma and Clinical Research Associates, receives royalties from APPI, Springer and an honoraria from Wiley. Alexandra Mogadam, Anne E. Keller, Jason P. Lerch and Elizabeth W. Pang report no conflicts of interest.

\section{Author details}

${ }^{1}$ Institute of Medical Science, Faculty of Medicine, University of Toronto, Toronto, Canada. ${ }^{2}$ Neurosciences and Mental Health, SickKids Research Institute, Toronto, Canada. ${ }^{3}$ Division of Neurology, Hospital for Sick Children, 555 University Avenue, Toronto M5G 1X8, Canada. ${ }^{4}$ Mathison Centre for Mental Health Research and Education, University of Calgary, Calgary, Canada. ${ }^{5}$ Genetics and Genome Biology, SickKids Research Institute, Toronto, Canada. ${ }^{6}$ Department of Psychiatry, Faculty of Medicine, University of Toronto, Toronto, Canada. ${ }^{7}$ Mouse Imaging Centre, Hospital for Sick Children, Toronto, Canada. ${ }^{8}$ Department of Medical Biophysics, Faculty of Medicine, University of Toronto, Toronto, Canada. ${ }^{9}$ Holland Bloorview Kids Rehabilitation Hospital, Toronto, Canada.
Received: 11 October 2018 Accepted: 29 July 2019

Published online: 19 August 2019

\section{References}

1. American Psychiatric Association. Diagnostic and statistical manual of mental disorders [Internet]. Fifth Edition: American Psychiatric Association; 2013.

2. Bishop D, Rutter M. Neurodevelopmental disorders: conceptual issues. In: Rutter's child and adolescent psychiatry. 5th ed. Hoboken: Blackwell Publishing Inc; 2008. p. 32-41.

3. World Health Organization. Multiaxial classification of child and adolescent psychiatric disorders: the ICD-10 classification of mental and behavioral disorders in children and adolescents. Cambridge: Cambridge University Press; 1996.

4. Leyfer OT, Folstein SE, Bacalman S, Davis NO, Dinh E, Morgan J, et al. Comorbid psychiatric disorders in children with autism: interview development and rates of disorders. J Autism Dev Disord. 2006;36(7):849-61.

5. Gazzellone MJ, Zarrei M, Burton CL, Walker S, Uddin M, Shaheen SM, et al. Uncovering obsessive-compulsive disorder risk genes in a pediatric cohort by high-resolution analysis of copy number variation. J Neurodev Disord. 2016;8:-36. https://doi.org/10.1186/s11689-016-9170-9.

6. Lionel AC, Crosbie J, Barbosa N, Goodale T, Thiruvahindrapuram B, Rickaby J, et al. Rare copy number variation discovery and cross-disorder comparisons identify risk genes for ADHD. Sci Transl Med. 2011;3(95):95ra75.

7. Lionel AC, Tammimies K, Vaags AK, Rosenfeld JA, Ahn JW, Merico D, et al. Disruption of the ASTN2/TRIM32 locus at 9q33.1 is a risk factor in males for autism spectrum disorders, ADHD and other neurodevelopmental phenotypes. Hum Mol Genet. 2014;23(10):2752-68. Available from: https:// academic.oup.com/hmg/article/23/10/2752/615766/Disruption-of-theASTN2-TRIM32-locus-at-9q33-1-is.

8. Ameis SH, Lerch JP, Taylor MJ, Lee W, Viviano JD, Pipitone J, et al. A diffusion tensor imaging study in children with ADHD, autism spectrum disorder, OCD, and matched controls: distinct and non-distinct white matter disruption and dimensional brain-behavior relationships. Am J Psychiatry. 2016;173(12):1213-22.

9. Baribeau DA, Doyle-Thomas KAR, Dupuis A, laboni A, Crosbie J, McGinn H, et al. Examining and comparing social perception abilities across childhoodonset neurodevelopmental disorders. J Am Acad Child Adolesc Psychiatry. 2015;54(6):479-86 e1.

10. Armbruster DJN, Ueltzhöffer K, Basten U, Fiebach CJ. Prefrontal cortical mechanisms underlying individual differences in cognitive flexibility and stability. J Cogn Neurosci. 2012;24(12):2385-99.

11. Dajani DR, Uddin LQ. Demystifying cognitive flexibility: implications for clinical and developmental neuroscience. Trends Neurosci. 2015;38(9):571-8.

12. Kim C, Cilles SE, Johnson NF, Gold BT. Domain general and domain preferential brain regions associated with different types of task switching: a meta-analysis. Hum Brain Mapp. 2012;33(1):130-42. Available from: http:// www.ncbi.nlm.nih.gov/pmc/articles/PMC3421461/.

13. Niendam TA, Laird AR, Ray KL, Dean YM, Glahn DC, Carter CS. Meta-analytic evidence for a superordinate cognitive control network subserving diverse executive functions. Cogn Affect Behav Neurosci. 2012;12(2):241-68.

14. Mogadam A, Keller AE, Taylor MJ, Lerch JP, Anagnostou E, Pang EW. Mental flexibility: an MEG investigation in typically developing children. Brain Cogn. 2017. Available from: http://www.sciencedirect.com/science/article/pii/S02 78262617301197.

15. Doesburg SM, Vidal J, Taylor MJ. Reduced theta connectivity during setshifting in children with autism. Front Hum Neurosci. 2013;7:785.

16. Taylor MJ, Donner EJ, Pang EW. fMRI and MEG in the study of typical and atypical cognitive development. Neurophysiol Clin Neurophysiol. 2012;42(12):19-25.

17. Velazquez JLP, Barcelo F, Hung Y, Leshchenko Y, Nenadovic V, Belkas $J$, et al. Decreased brain coordinated activity in autism spectrum disorders during executive tasks: reduced long-range synchronization in the fronto-parietal networks. Int J Psychophysiol. 2009;73(3):341-9.

18. D'Cruz A-M, Mosconi MW, Ragozzino ME, Cook EH, Sweeney JA. Alterations in the functional neural circuitry supporting flexible choice behavior in autism spectrum disorders. Transl Psychiatry. 2016;6(10):e916. Available from: https://www.ncbi.nlm.nih.gov/pmc/articles/PMC5315543/.

19. Rubia K, Cubillo A, Smith AB, Woolley J, Heyman I, Brammer MJ. Disorderspecific dysfunction in right inferior prefrontal cortex during two inhibition 
tasks in boys with attention-deficit hyperactivity disorder compared to boys with obsessive-compulsive disorder. Hum Brain Mapp. 2010;31(2):287-99.

20. Rubia K, Halari R, Cubillo A, Mohammad A-M, Scott S, Brammer M. Disorderspecific inferior prefrontal hypofunction in boys with pure attention-deficit/ hyperactivity disorder compared to boys with pure conduct disorder during cognitive flexibility. Hum Brain Mapp. 2010;31(12):1823-33.

21. Smith AB, Taylor E, Brammer M, Toone B, Rubia K. Task-specific hypoactivation in prefrontal and temporoparietal brain regions during motor inhibition and task switching in medication-naive children and adolescents with attention deficit hyperactivity disorder. Am J Psychiatry. 2006;163(6):1044-51.

22. Britton JC, Rauch SL, Rosso IM, Killgore WDS, Price LM, Ragan J, et al. Cognitive inflexibility and frontal-cortical activation in pediatric obsessivecompulsive disorder. J Am Acad Child Adolesc Psychiatry. 2010;49(9):944-53.

23. Hansen PC, Kringelbach ML, Salmelin R. Introduction. In: Hansen P, Kringelbach ML, Salmelin R, editors. MEG: an introduction to methods. Oxford, New York: Oxford University Press; 2010. p. vii-x.

24. Hari R, Salmelin R. Magnetoencephalography: from SQUIDs to neuroscience: Neuroimage 20th anniversary special edition. Neurolmage. 2012;61(2):386-96.

25. Mišić B, Doesburg SM, Fatima Z, Vidal J, Vakorin VA, Taylor MJ, et al. Coordinated information generation and mental flexibility: large-scale network disruption in children with autism. Cereb Cortex. 2015;25(9): 2815-27.

26. Ickowicz A, Schachar RJ, Sugarman R, Chen SX, Millette C, Cook L. The parent interview for child symptoms: a situation-specific clinical research interview for attention-deficit hyperactivity and related disorders. Can J Psychiatry. 2006;51(5):325-8. https://doi.org/10.1177/070674370605100508.

27. Scahill L, Riddle MA, McSwiggin-Hardin M, Ort SI, King RA, Goodman WK, et al. Children's Yale-Brown obsessive compulsive scale: reliability and validity. J Am Acad Child Adolesc Psychiatry. 1997;36(6):844-52.

28. Bodfish JW, Symons FJ, Parker DE, Lewis MH. Varieties of repetitive behavior in autism: comparisons to mental retardation. J Autism Dev Disord. 2000; 30(3):237-43.

29. Bodfish JW, Symons FJ, Lewis MH. The Repetitive Behavior Scale: a test manual. Morganton: Western Carolina Center Research Reports; 1999.

30. Lord C, Risi S, Lambrecht L, Cook EH, Leventhal BL, DiLavore PC, et al. The Autism Diagnostic Observation Schedule-Generic: a standard measure of social and communication deficits associated with the spectrum of autism. J Autism Dev Disord. 2000;30(3):205-23.

31. Lord C, Rutter M, Couteur AL. Autism Diagnostic Interview-Revised: revised version of a diagnostic interview for caregivers of individuals with possible pervasive developmental disorders. J Autism Dev Disord. 1994;24(5):659-85.

32. Park LS, Burton CL, Dupuis A, Shan J, Storch EA, Crosbie J, et al. The Toronto Obsessive-Compulsive Scale: psychometrics of a dimensional measure of obsessive-compulsive traits. J Am Acad Child Adolesc Psychiatry. 2016;55(4): 310-8 e4.

33. Swanson JM, Schuck S, Porter MM, Carlson C, Hartman CA, Sergeant JA, et al. Categorical and dimensional definitions and evaluations of symptoms of ADHD: history of the SNAP and the SWAN Rating Scales. Int J Educ Psychol Assess. 2012;10(1):51-70. Available from: http://www.ncbi.nlm.nih. gov/pmc/articles/PMC4618695/.

34. Oh A, Vidal J, Taylor MJ, Pang EW. Neuromagnetic correlates of intra- and extra-dimensional set-shifting. Brain Cogn. 2014;86:90-7.

35. Statistical Parametric Mapping. London: Wellcome Trust Centre for Neuroimaging. 2014.

36. Oostenveld R, Fries P, Maris E, Schoffelen J-M. FieldTrip: open source software for advanced analysis of MEG, EEG, and invasive electrophysiological data. Comput Intell Neurosci. 2010;2011:e156869. Available from: https:/www. hindawi.com/journals/cin/2011/156869/abs/.

37. Delorme A, Makeig S. EEGLAB: an open source toolbox for analysis of single-trial EEG dynamics including independent component analysis. J Neurosci Methods. 2004;134(1):9-21.

38. Kovacevic N, Mclntosh AR. Groupwise independent component decomposition of EEG data and partial least square analysis. Neurolmage. 2007;35(3):1103-12.

39. Friston K, Henson R, Phillips C, Mattout J. Bayesian estimation of evoked and induced responses. Hum Brain Mapp. 2006;27(9):722-35.

40. Mattout J, Phillips C, Penny WD, Rugg MD, Friston KJ. MEG source localization under multiple constraints: an extended Bayesian framework. Neurolmage. 2006;30(3):753-67.
41. Brookes MJ, Hall EL, Robson SE, Price D, Palaniyappan L, Liddle EB, et al. Complexity measures in magnetoencephalography: measuring "disorder" in schizophrenia. PLOS ONE. 2015;10(4):e0120991. Available from: http:// journals.plos.org/plosone/article?id=10.1371/journal.pone.0120991.

42. Rorden C, Karnath H-O, Bonilha L. Improving lesion-symptom mapping. J Cogn Neurosci. 2007;19(7):1081-8.

43. Penny WD, Holmes AP, Friston KJ. Random effects analysis. In: Human brain function. 2nd ed; 2003. p. 843-50.

44. Gogtay N, Giedd JN, Lusk L, Hayashi KM, Greenstein D, Vaituzis AC, et al. Dynamic mapping of human cortical development during childhood through early adulthood. Proc Natl Acad Sci U S A. 2004;101(21):8174-9. Available from: http://www.ncbi.n/m.nih.gov/pmc/articles/PMC419576/.

45. Sowell ER, Peterson BS, Thompson PM, Welcome SE, Henkenius AL, Toga AW. Mapping cortical change across the human life span. Nat Neurosci. 2003:6(3):309-15.

46. Bunge SA, Dudukovic NM, Thomason ME, Vaidya CJ, Gabrieli JDE. Immature frontal lobe contributions to cognitive control in children. Neuron. 2002; 33(2):301-11. Available from: http://www.cell.com/neuron/abstract/S0896-62 73(01)00583-9.

47. Casey BJ, Galvan A, Hare TA. Changes in cerebral functional organization during cognitive development. Curr Opin Neurobiol. 2005;15(2):239-44.

48. Crone EA, Zanolie K, Leijenhorst LV, Westenberg PM, Rombouts SARB. Neural mechanisms supporting flexible performance adjustment during development. Cogn Affect Behav Neurosci. 2008;8(2):165-77. Available from: http://link.springer.com/article/10.3758/CABN.8.2.165.

49. Rubia K, Smith AB, Woolley J, Nosarti C, Heyman I, Taylor E, et al. Progressive increase of frontostriatal brain activation from childhood to adulthood during event-related tasks of cognitive control. Hum Brain Mapp. 2006; 27(12):973-93.

50. Booth JR, Burman DD, Meyer JR, Lei Z, Trommer BL, Davenport ND, et al. Larger deficits in brain networks for response inhibition than for visual selective attention in attention deficit hyperactivity disorder (ADHD). J Child Psychol Psychiatry. 2005;46(1):94-111.

51. Cherkasova MV, Hechtman L. Neuroimaging in attention-deficit hyperactivity disorder: beyond the frontostriatal circuitry. Can J Psychiatry Rev Can Psychiatr. 2009;54(10):651-64.

52. Cubillo A, Halari R, Ecker C, Giampietro V, Taylor E, Rubia K. Reduced activation and inter-regional functional connectivity of fronto-striatal networks in adults with childhood attention-deficit hyperactivity disorder (ADHD) and persisting symptoms during tasks of motor inhibition and cognitive switching. J Psychiatr Res. 2010;44(10):629-39.

53. Dickstein SG, Bannon K, Castellanos FX, Milham MP. The neural correlates of attention deficit hyperactivity disorder: an ALE meta-analysis. J Child Psycho Psychiatry. 2006;47(10):1051-62.

54. Rubia K, Overmeyer S, Taylor E, Brammer M, Williams SCR, Simmons A, et al. Hypofrontality in attention deficit hyperactivity disorder during higher-order motor control: a study with functional MRI. Am J Psychiatry. 1999;156(6):891-6.

55. Fitzgerald KD, Stern ER, Angstadt M, Nicholson-Muth KC, Maynor MR, Welsh $\mathrm{RC}$, et al. Altered function and connectivity of the medial frontal cortex in pediatric obsessive-compulsive disorder. Biol Psychiatry. 2010;68(11):1039-47.

56. Aylward EH, Harris GJ, Hoehn-Saric R, Barta PE, Machlin SR, Pearlson GD. Normal caudate nucleus in obsessive-compulsive disorder assessed by quantitative neuroimaging. Arch Gen Psychiatry. 1996;53(7):577-84.

57. Menzies L, Chamberlain SR, Laird AR, Thelen SM, Sahakian BJ, Bullmore $E T$. Integrating evidence from neuroimaging and neuropsychological studies of obsessive-compulsive disorder: the orbitofronto-striatal model revisited. Neurosci Biobehav Rev. 2008;32(3):525-49.

58. Chamberlain SR, Menzies L, Hampshire A, Suckling J, Fineberg NA, del Campo N, et al. Orbitofrontal dysfunction in patients with obsessivecompulsive disorder and their unaffected relatives. Science. 2008; 321(5887):421-2.

59. Gu B-M, Park J-Y, Kang D-H, Lee SJ, Yoo SY, Jo HJ, et al. Neural correlates of cognitive inflexibility during task-switching in obsessive-compulsive disorder. Brain. 2008;131(1):155-64 Available from: http://brain.oxfordjournals.org/ content/131/1/155

60. Schmitz N, Rubia K, Daly E, Smith A, Williams S, Murphy DGM. Neural correlates of executive function in autistic spectrum disorders. Biol Psychiatry. 2006:59(1):7-16.

61. Ecker C, Bookheimer SY, Murphy DGM. Neuroimaging in autism spectrum disorder: brain structure and function across the lifespan. Lancet Neurol. 2015;14(11):1121-34 
62. Keown CL, Shih $\mathrm{P}$, Nair A, Peterson N, Mulvey ME, Müller R-A. Local functional overconnectivity in posterior brain regions is associated with symptom severity in autism spectrum disorders. Cell Rep. 2013;5(3):567-72. Available from: http://www.sciencedirect.com/science/article/pii/S2211124 71300572X

63. Maximo JO, Keown CL, Nair A, Müller R-A. Approaches to local connectivity in autism using resting state functional connectivity MRI. Front Hum Neurosci. 2013;7. Available from: http://journal.frontiersin.org/article/10.3389/ fnhum.2013.00605/full.

64. Zeeland AAS-V, Abrahams BS, Alvarez-Retuerto Al, Sonnenblick LI, Rudie JD, Ghahremani D, et al. Altered functional connectivity in frontal lobe circuits is associated with variation in the autism risk gene CNTNAP2. Sci Transl Med. 2010;2(56):56ra80

65. D'Cruz A-M, Ragozzino ME, Mosconi MW, Shrestha S, Cook EH, Sweeney JA. Reduced behavioral flexibility in autism spectrum disorders. Neuropsychology. 2013;27(2):152-60.

66. Miller HL, Ragozzino ME, Cook EH, Sweeney JA, Mosconi MW. Cognitive set shifting deficits and their relationship to repetitive behaviors in autism spectrum disorder. J Autism Dev Disord. 2015;45(3):805-15.

67. Haber SN. Corticostriatal circuitry. Dialogues Clin Neurosci. 2016;18(1):7-21. Available from: http://www.ncbi.nlm.nih.gov/pmc/articles/PMC4826773/.

68. Middleton FA, Strick PL. Basal ganglia output and cognition: evidence from anatomical, behavioral, and clinical studies. Brain Cogn. 2000;42(2):183-200.

69. Langen M, Durston S, Kas MJH, van Engeland H, Staal WG. The neurobiology of repetitive behavior: ... and men. Neurosci Biobehav Rev. 2011;35(3):356-65.

70. Jarbo K, Verstynen TD. Converging structural and functional connectivity of orbitofrontal, dorsolateral prefrontal, and posterior parietal cortex in the human striatum. J Neurosci. 2015;35(9):3865-78.

71. Alexander GE, DeLong MR, Strick PL. Parallel organization of functionally segregated circuits linking basal ganglia and cortex. Annu Rev Neurosci. 1986:9(1):357-81. Available from: https://www.annualreviews.org/doi/1 0.1146/annurev.ne.09.030186.002041.

72. Osmon DC, Smerz JM. Neuropsychological evaluation in the diagnosis and treatment of Tourette's syndrome. Behav Modif. 2005;29(5):746-83.

73. Hollander E, Anagnostou E, Chaplin W, Esposito K, Haznedar MM, Licalzi E, et al. Striatal volume on magnetic resonance imaging and repetitive behaviors in autism. Biol Psychiatry. 2005;58(3):226-32.

74. Langen M, Bos D, Noordermeer SDS, Nederveen $H$, van Engeland $H$, Durston S. Changes in the development of striatum are involved in repetitive behavior in autism. Biol Psychiatry. 2014;76(5):405-11.

75. Rojas DC, Peterson E, Winterrowd E, Reite ML, Rogers SJ, Tregellas JR. Regional gray matter volumetric changes in autism associated with social and repetitive behavior symptoms. BMC Psychiatry. 2006;6:56. https://doi. org/10.1186/1471-244X-6-56.

76. Chiang H-L, Chen Y-J, Shang C-Y, Tseng W-Yl, Gau SS-F. Different neural substrates for executive functions in youths with ADHD: a diffusion spectrum imaging tractography study. Psychol Med Camb. 2016:46(6):1225-38.

77. Akkermans SEA, Rheinheimer N, Bruchhage MMK, Durston S, Brandeis D, Banaschewski T, et al. Frontostriatal functional connectivity correlates with repetitive behaviour across autism spectrum disorder and obsessivecompulsive disorder. Psychol Med. 2018;26:1-9.

78. Vaghi MM, Vértes PE, Kitzbichler MG, Apergis-Schoute AM, van der Flier $\mathrm{FE}$, Fineberg NA, et al. Specific frontostriatal circuits for impaired cognitive flexibility and goal-directed planning in obsessive-compulsive disorder: evidence from resting-state functional connectivity. Biol Psychiatry. 2017;81(8):708-17.

79. Jacquemont S, Coe BP, Hersch M, Duyzend MH, Krumm N, Bergmann S, et al. A higher mutational burden in females supports a "female protective model" in neurodevelopmental disorders. Am J Hum Genet. 2014;94(3):415-25. Available from: http://www.sciencedirect.com/science/article/pii/S0002929714 000597.

80. Valera EM, Brown A, Biederman J, Faraone SV, Makris N, Monuteaux MC, et al. Sex differences in the functional neuroanatomy of working memory in adults with ADHD. Am J Psychiatry. 2010;167(1):86-94.

81. Werling DM, Geschwind DH. Sex differences in autism spectrum disorders. Curr Opin Neurol Curr Opin Neurol. 2013;26(2):146-53.

\section{Publisher's Note}

Springer Nature remains neutral with regard to jurisdictional claims in published maps and institutional affiliations.

Ready to submit your research? Choose BMC and benefit from:

- fast, convenient online submission

- thorough peer review by experienced researchers in your field

- rapid publication on acceptance

- support for research data, including large and complex data types

- gold Open Access which fosters wider collaboration and increased citations

- maximum visibility for your research: over $100 \mathrm{M}$ website views per year

At BMC, research is always in progress.

Learn more biomedcentral.com/submissions 